Comparison of Lidar building point cloud with reference model for deep comprehension of cloud structure

F. Tarsha Kurdi*, M. Awrangjeb

Institute for Integrated and Intelligent Systems, Griffith University, Nathan QLD 4111, Australia

(f.tarshakurdi, m.awrangjeb)@ griffith.edu.au 


\title{
Comparison of Lidar building point cloud with reference model for deep comprehension of cloud structure
}

\begin{abstract}
This paper studies the fidelity level of the extracted Lidar (LIght Detection And Ranging) building point cloud in relation to the original building. In this context, the building point cloud is compared with a reference model. This comparison allows a deep understanding of the point cloud structure with respect to both the actual building and the constructed model. Consequently, the source of the incompatibility in a (reference or constructed) building model is determined and described. Thus, this study considers four aspects of the building point cloud. First, the errors of building point cloud extraction and the undesirable points are quantified. Hence, it is found that the percentages of undesirable points are sometimes considerable (between 15\% and 70\%). Second, the evaluation of the fit of the altimetry with the reference model shows that the roof plane equations calculated from Lidar data can be more precise than those of reference model, Third, it is noted that the segmentability level between different point densities and building typologies are variable. Finally, the per plane comparison mentions the incompatibility of the plane boundaries of point cloud with reference model. Moreover, considerable differences are noted between the theoretical and the true point densities.
\end{abstract}

Key words: Lidar; modelling; segmentation; differences analysis; accuracy; point cloud

\section{Introduction}

A typical Lidar (Light detection and ranging) system consists of three main elements, a GNSS (Global Navigation Satellite System) to provide the 3-dimensional (3D) aircraft position, an INS (Inertial Navigation System) to measure the aircraft orientation and acceleration, and a laser unit to provide distance information from the laser beam source point to the objects on the ground (Habib 2018). Airborne laser scanning is achieved in several parallel strips with overlapping areas. The adjustment of these strips by controlling the overlapping areas serves to generate a 3D point cloud with homogeneous accuracy (Toth and Koppanyi 2018). The accuracy of Lidar point cloud is related to three 
factors, which are the accuracy of laser system components, the calibration of the laser system, and the adjustment of scan strips (Wehr 2018).

The next envisaged task is the processing of laser data. In urban areas, the extraction of building class from the city scene can be achieved automatically or manually before starting the modelling stage. In the context of automatic building modelling, two major approaches are presented in the literature, the model driven or parametric approach, and the data driven or non-parametric approach (Shan et al. 2018 and Tarsha Kurdi et al. 2007). The majority of existing modelling algorithms generate differences because of noise presence in a building point cloud data. At this stage, it is noted that the building point cloud is sometimes irregular heterogeneous due to the scanning technique accuracy (Zhang et al. 2018). All these reasons also generate incompatibilities at the level of the constructed 3D building model.

Thus, according to Tarsha Kurdi and Awrangjeb (2020), the building model suffers from accumulation of two types of incompatibility: the first one is due to the infidelity of point cloud with the original building. The employed modelling algorithm generates the second type of incompatibility. That is why estimating the accuracy of the constructed building model can not be avoided. In this context, the accuracy estimation methods suggested in the literature can be classified into two categories. The first one uses the point cloud as a reference model such as Akca et al. (2010), Dorninger and Pfeifer (2008), Erberink and Vossleman (2011), Ostrowski et al. (2018), Park et al. (2019) and Sampath and Shan (2010). The approaches of the second category employ 3D reference models calculated from areal images or reference measurements in the field (Gülch, et al. 2018, Jung and Sohn, 2018, Rottensteiner et al. 2014 and Kaartinen et al. 2005).

With regard to the presence of the last two approaches of accuracy estimation, the 
estimated accuracy of the calculated 3D building model will be related to the quality of the adopted reference model in addition to the employed accuracy indices (Ostrowski et al. 2018). Furthermore, the accuracy analysis of the point cloud in addition to the calculated building model can help to determine the sources, quality, quantity, and the handling methods of model differences. Consequently, the selection of the accuracy estimation approach in addition to the reference model represents a real challenge. With regard to the selection of the suitable accuracy estimation approach, five questions arise:

(1) What is the fidelity level of the extracted building point cloud in relation to the original building?

(2) To what extent are the cloud differences generated by the laser scanning system and by the extraction operation?

(3) How much are the building model differences generated only by the modelling algorithm?

(4) How much are the building model differences generated by the modelling algorithm and the laser scanning system together?

(5) What are the types of the differences that are expressed by different accuracy indices suggested in the literature?

This paper contributes to a greater understanding of the building point cloud structure in comparison to the original building, therfore it focuses on the first and second questions. In future research, the other three questions will be studied.

\section{Related work}

In the field of Lidar data processing, the construction of 3D building models must be followed by accuracy estimation. In order to achieve this, a reference model is required. According to ISO (International Organization for Standardization) (2005), the definition 
of data quality is the degree to which a set of inherent characteristics are fulfilled. With regard to the incompetence in the project management practices, several Architecture, Engineering, Construction and Facilities Management (AEC/FM) associations realized the Building Information Modelling (BIM) (Edirisinghe and London 2015). Hence, the reference model (BIM-CAREM) for controlling the BIM competence of AEC/FM processes, consists of two parts which are the BIM process reference model and the BIM measurement framework (Yilmaz et al. 2019). Unfortunately, there are no standard approaches to measure the 3D model calculated from Lidar data quality (Stoter et al. 2016 and Wong and Ellul 2016). Moreover, the absence of Lidar data and BIM regulations was the motivation for suggesting the employment of the Lidar data evaluation concept in the procedure of developing a 3D-BIM model based on Lidar point clouds (Warchol 2019).

In this context, geographic data quality may be exposed by multiple factors such as completeness, logical consistency, positional accuracy, thematic accuracy, temporal quality and usability element (ISO 2013 and Warchol 2019). According to Zhao et al. (2014), there are four main sources of error within 3D city models: choice of modelling tools, model optimization, conversion and semantics editing. However, in order to estimate the accuracy of a 3D building model calculated from the Lidar point cloud, two principal tests have to be carried out. The first test is the location accuracy of the 3D building model in the 3D space, while the second is the accuracy of geometric form of the building model.

The location accuracy value of the $3 \mathrm{D}$ building model is related to the position of the original point cloud and to the noise within the point cloud (Akca et al. 2010). On the one hand, if the building point cloud suffers from systematic error, the location and/or the orientation of the constructed model will be shifted. On the other hand, it is sometimes hard to determine the separation between the building and terrain due to the heterogenic 
density and distribution of the point cloud. There may also be the presence of noise, vegetation, and obstacles between the building and the terrain. That is why the modelling algorithm may generate a vertical shift in the position of the building model. In this context, Guptill and Morrison (1995) and Krämer et al. (2007) classify the positional accuracy into two classes: the horizontal and the vertical accuracies. Akca et al. (2010) and Ostrowski et al. (2018) suggest that the global positional accuracy (the position and the orientation) of the calculated 3D building model must be verified.

Concerning the accuracy of geometric form of the building model, Cheng et al. (2015) and Zhang et al. (2018) classify the quality control of the calculated 3D models into two main tasks: examining the visual effects and assessing the geometric accuracy which is also named parametric accuracy.

In the context of the visual accuracy estimation, Zhang et al. (2018) refer to the visual estimation of the accuracy using a qualitative method, which checks the quality of estimated dominant directions through visual comparison. For this purpose, they use reference data such as maps, aerial photographs and Digital Terrain Model (DTM) interpolated from ground measurements. In the same context, Cheng et al. (2015) conducted a visual quality estimation by inviting 100 participants to vote about the quality of building models. Thereafter, based on the fuzzy set theory and information entropy method, a fuzzy realistic index is calculated for each building model to evaluate the visual effects by linking the subjective experience with the quality of the 3D models.

In the context of the geometric accuracy (parametric accuracy), Cheng et al. (2015) and Ostrowski et al. (2018) summarize the accuracy estimation approaches of the literature into two classes. In the first class (feature-based), a reference model is required in order to compare the calculated model with the reference, whereas, in the second class (Lidar- 
based), the Lidar point cloud is considered as reference data. The accuracy estimation methods in these two approaches are reviewed below.

\subsection{Feature-based accuracy}

In the feature-based estimation accuracy approach, a reference model is required in order to compare the calculated model with the reference. In literature, several methods are suggested for constructing the reference model. In this context, Shan et al. (2018) derive the reference model by manual segmentation of the building point cloud. Rottensteiner and Clode (2018) generate the reference model in a semiautomatic working environment by using digital aerial images and Lidar data. Jung and Sohn (2018) and Tarsha Kurdi et al. (2007) use 3D rooftop models reconstructed by the manual stereo plotting method, while Jung and Sohn (2018) use the International Society for Photogrammetry and Remote Sensing (ISPRS) benchmark datasets (Rottensteiner et al. 2014). Zhang et al. (2018) employ several reference data together: the maps, the aerial photographs and the DTM interpolated from ground measurements. Cheng et al. (2015) and Demir (2018) employ a reference model constructed from field survey. Awrangjeb et al. (2018) employed 2D reference data collected through monoscopic image measurement in addition to 3D reference data collected at an earlier date.

In addition to the above methods of constructing the reference model, the EuroSDR (www.eurosdr.net) uses three types of reference data (Gülch, et al. 2018 and Kaartinen et al. 2005):

- Reference data (collected using a tachometer) include corners of walls, roofs, chimneys and equivalent constructions, and ground points at building corners.

- Reference data include roof points measured manually from aerial images. 
- Reference raster images of building ground plans produced from cadastral maps after some minor updating and refinements based on reference measurements in the field.

Once the reference model is available, the accuracy estimation may be carried out by calculating the differences between the features of the constructed model and those of the reference model. For this purpose, three main roof features are considered: planes, boundaries and vertices. The suggested accuracy tests for each feature type are presented in the following sections.

\subsubsection{Roof planes}

Awrangjeb et al. (2018) employ two classes of evaluation metrics in order to estimate the accuracy of the extracted roof planes: object-based and pixel-based. In the two classes, they employ the confusion matrix in two different ways. The object-based evaluation counts the number of objects, whereas the pixel-based evaluation counts the number of pixels in the objects. They found that the object-based evaluation gives higher results than the pixel-based evaluation. Indeed, the quality is $10 \%$ better than the latter evaluation. Furthermore, the RMSE (Root Mean Square Error) is also used in both the 2D and the 3D spaces to evaluate the geometric accuracy.

Shan et al. (2018) calculate six index-values for evaluating the accuracy of the constructed 3D building model: completeness, correctness, detection cross-lap rate, reference crosslap rate, boundary precision, and boundary recall. Thus, all results were nearly $90 \%$. At this stage, it is useful to mention that some index-values can be calculated directly from the confusion matrix or the error matrix, which is widely employed in remote sensing domain (Bossler et al. 2010 and Demir 2018). Then, using the confusion matrix, the quantitative values for completeness, correctness and quality criteria can be assigned 
(Jung and Sohn 2018). However, the use of the confusion matrix suggested by Shan et al. (2018) is based on the comparison of the number of roof planes instead of the number of pixels. Moreover, according to an accuracy viewpoint, they classify the building roof planes into following classes: missed planes where an actual plane is not segmented, spurious planes, which may be either oversegmented planes or undersegmented planes and correctly detected planes.

\subsubsection{Plane boundaries and roof vertices}

Demir (2018), Gülch et al. (2018) and Kaartinen et al. (2005) estimate the building model accuracy by calculating the average absolute distance differences of building model length and roof inclination. Jung and Sohn (2018) and Rottensteiner and Clode (2018) estimate the constructed model accuracy by calculating the RMSE values of the coordinate differences of corresponding vertices in the reconstruction results and the reference data. The average values of RMSE are between $1.25 \mathrm{~m}$ and $0.5 \mathrm{~m}$. Demir (2018) compares the shifts in $X, Y$ and $Z$ coordinates of roof vertices.

\subsection{Lidar-based Accuracy}

Akca et al. (2010), Dorninger and Pfeifer (2008), Erberink and Vossleman (2011), Ostrowski et al. (2018), Park et al. (2019), Sampath and Shan (2010), Tarsha Kurdi (2008) and Tarsha Kurdi et al. (2019) suggest using the Lidar point cloud as the reference data. Consequently, one of the crucial elements of their approaches of modelling accuracy estimation is the calculation of distances (point to point) between the $3 \mathrm{D}$ building model and the point cloud. There are two main forms to present the accuracy estimation result: vector form (Ostrowski et al. 2018) and raster form (Tarsha Kurdi 2008). Moreover, Sampath and Shan (2010) list the minimum, maximum, mean, and standard deviation of three measurements which are the triangle edge lengths for all the roof segments, the 
distances of a segmented point from its corresponding plane and the differences (in degrees) of the normal vectors with respect to their corresponding cluster centers. In the same context, Park et al. (2019) extract model key points, which represent the roof corners. Then, these points are considered as reference models that are used for estimating the accuracy of constructed building models by calculating the root mean square values in addition to standard deviation.

\section{Data}

In this study, three datasets are used (see Figure 1). The characteristics of these datasets are shown in Table 1. The first point cloud is of the city of Strasbourg in France. Several zones with different typology natures are covered. However, point density and accuracy are lower in comparison to the second dataset, which was captured over the city of Vaihingen in Germany (Cramer 2010). A few residential buildings, historic buildings, and small-detached houses that are surrounded by trees characterize this area. The third point cloud is of Toronto city (Cramer 2010). It contains representative scene characteristics of a modern mega city in Canada including a mixture of low- and highstorey buildings with a wide variety of rooftop structures, streets and roads. Finally, it is important to note that only the first returns of laser beams are employed in this study.

Table 1. Characteristics of the three datasets used in this paper.

\begin{tabular}{|c|c|c|c|}
\hline & Toronto & Strasbourg & Vaihingen \\
\hline Acquisition & February 2009 & September 2004 & 21 August 2008 \\
\hline Sensor & $\begin{array}{c}\text { Optech ALTM- } \\
\text { ORION M }\end{array}$ & $\begin{array}{c}\text { TopScan (Optech } \\
\text { ALTM 1225) }\end{array}$ & $\begin{array}{c}\text { Leica Geosystems } \\
\text { Leica ALS50 system }\end{array}$ \\
\hline $\begin{array}{c}\text { Points density } \\
\left.\text { (points } / \mathrm{m}^{2}\right)\end{array}$ & 6 & 1.3 & $4-6.7$ \\
\hline Flight height $(\mathrm{m})$ & 650 & 1440 & 500 \\
\hline
\end{tabular}




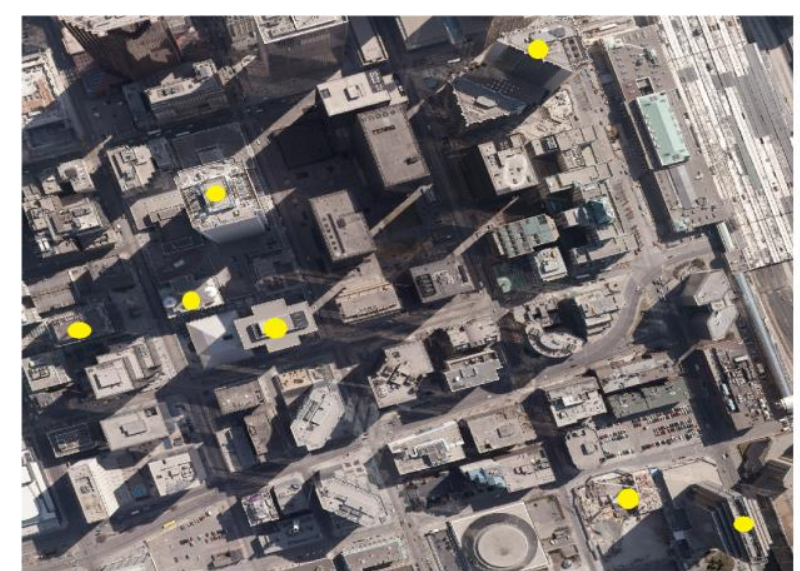

(a)

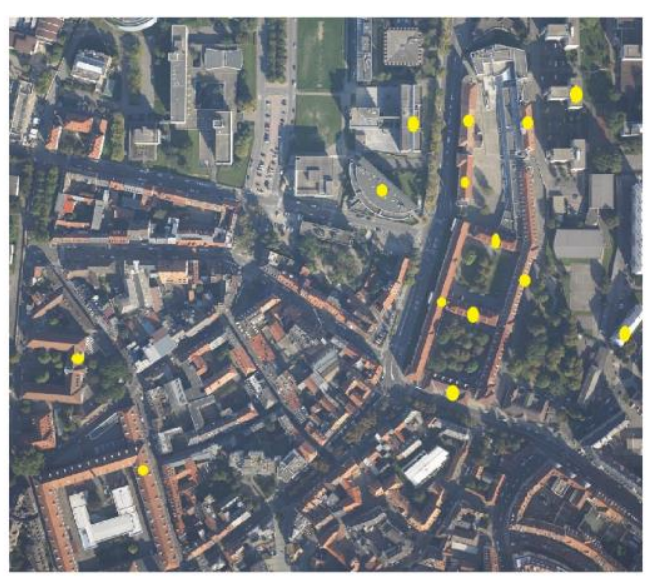

(b)

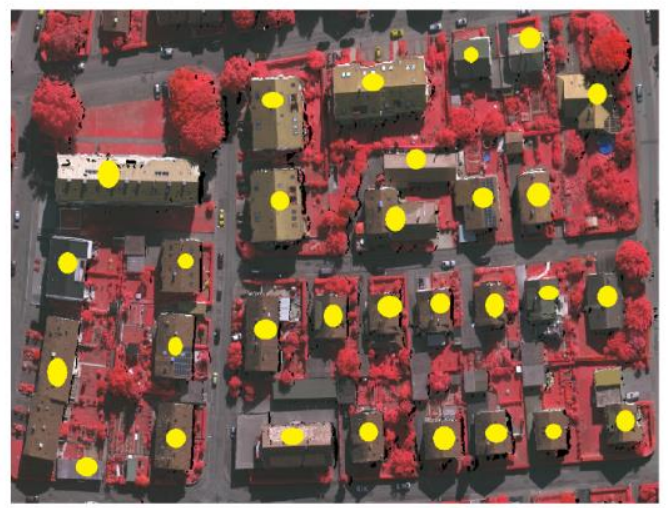

(c)

Figure 1. Aerial photos of three datasets used in this paper. Tested zones presented in Table 3; (a) Toronto; (b) Strasbourg; (c) Vaihingen; Tested building point clouds are marked by yellow circles.

\section{Comparison of building point cloud to reference model}

There are three models of each building: the original building, the point cloud, and the constructed model from the building point cloud. As discussed in Section 2, there are two main approaches to evaluate the accuracy of geometric form of the calculated model. The first approach suggests comparing the calculated model with the reference model whereas the second approach considers the building point cloud as a reference model (Cheng et al. 2015 and Ostrowski et al. 2018). It is important to note that the reference model is calculated starting from the original model (the scanned building). Therefore, regardless 
the scale factor, the original model and the reference model are similar but not exactly identical. Despite the difference between the two models, the reference model is still the most faithful model to the original model, which is why it may be considered a prototype. The calculated model has to be faithful to the building point cloud because the point cloud is the input data for constructing this model. Moreover, the point cloud has to be faithful to the reference and to the original models because it represents the calculated model from the original building. Consequently, the building point cloud plays three roles simultaneously: the role of the calculated model from the original model, the role of the reference model of the calculated building model and the role of input data for the calculated model. The comparison of the building point cloud to the reference model allows determining the harmonization level between the building point cloud and the reference model. This comparison permits also to measure the differences that the point cloud suffers from.

This paper suggests a new approach for comparing the building point cloud with the reference model. The organigram presented in Figure 2 illustrates the comparison steps in addition to the relationships between them. There are five main steps are discussed as follows:

(1) The estimation of the extraction error of the building point cloud from the entire scanned scene point cloud,

(2) The estimation of the quantity of undesirable points in the detected building point cloud,

(3) The evaluation of the altimetry fitting level of the building point cloud to the reference model,

(4) The segmentability of the roof point cloud, and

(5) The per plane comparison between the point cloud and the reference model. 


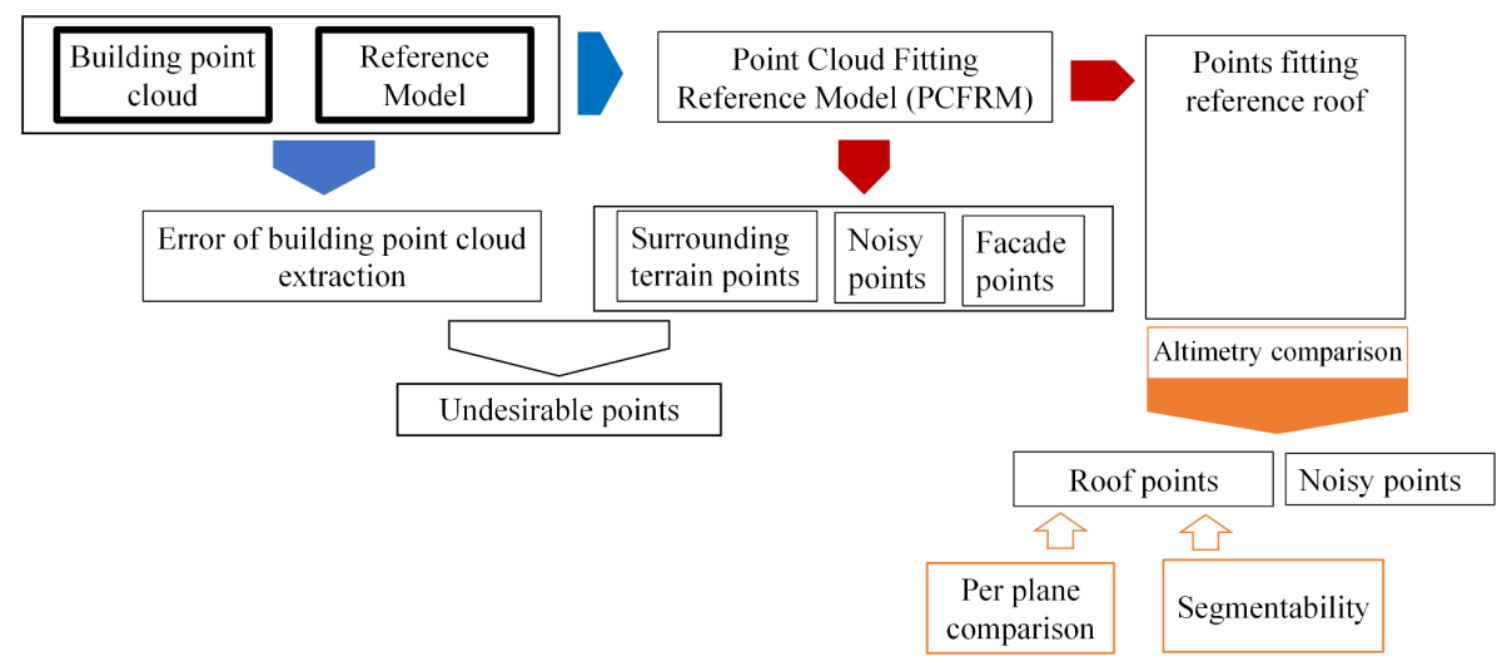

Figure 2. Comparison steps of building point cloud with reference model.

\subsection{Error of building point cloud extraction}

The airborne laser scanning in urban areas measures a 3D point cloud covering the scanned city. In order to model any given building in the scanned city, this building point cloud first has to be extracted from the global point cloud. In order to achieve this purpose, two main ways are envisaged: automatic extraction and manual extraction. Despite the manual extraction being very slow in comparison to the automatic extraction, it is still more accurate than the automatic extraction, especially if an expert in the Lidar data processing field performs it. Indeed, in the first way, the extraction is carried out per point basis and the operator may have extra information about the scanned scene such as aerial photos, city maps and/or other collected information, in addition to supplementary informatics navigation and visualization tools. That is why the manual extraction is adopted in this study.

In this research, two experiments are carried out. In the first one, two buildings from Vaihingen point cloud are selected in order to be tested manually by three skilled operators (see Figure 3). In the second experiment, several buildings from each tested site are selected in order to be tested manually by only one skilled operator (see Figure 1). 
The reference models of all selected buildings in the two categories are available. The involved skilled operators are experts in the Lidar data processing field who extract the point clouds of the selected buildings manually. The reference models are reconstructed by the manual stereo plotting method (Rottensteiner et al. 2014). A building point cloud is a set of points that belongs to the same building. This definition is adopted for extracting the selected building point clouds from the city cloud.

It is noted in the first experiment that the three point clouds extracted by different operators of each of the two selected buildings are not identical (Table 2). This difference between the point clouds extracted for the same building may be explained by the presence of confusing points in the connection zone between terrain, vegetation and other objects surrounding the building and the building itself. Moreover, the detail points attached to building facades are sometimes confused. 


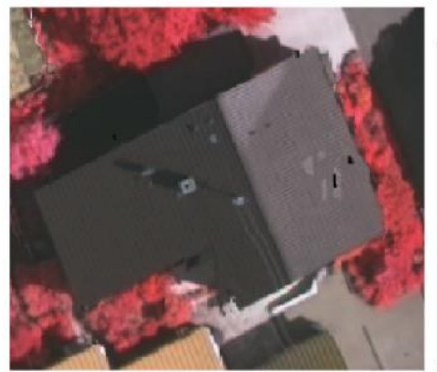

(a)

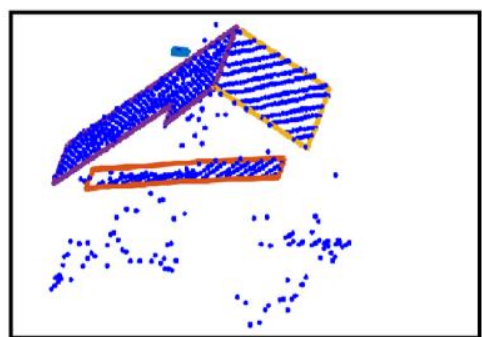

(d)

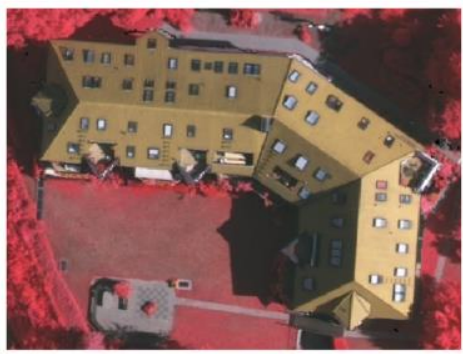

(g)

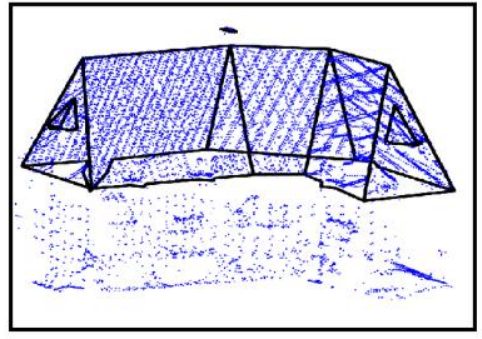

(j)

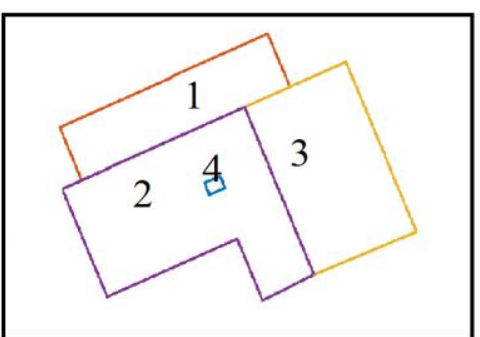

(b)

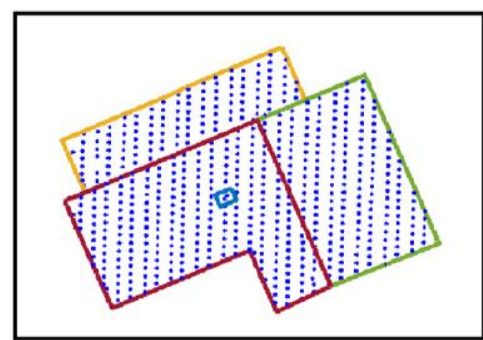

(e)

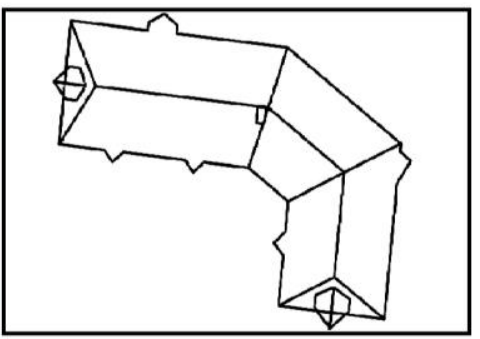

(h)

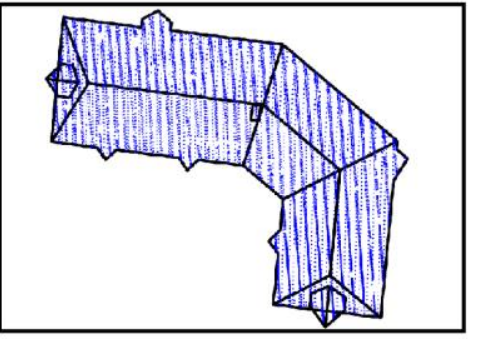

(k)

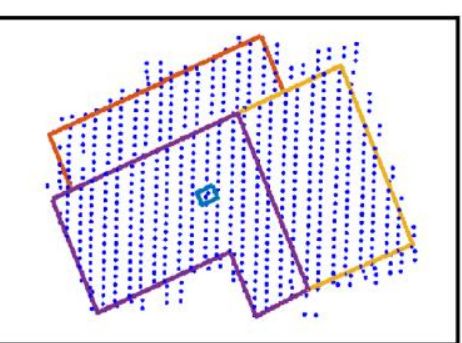

(c)

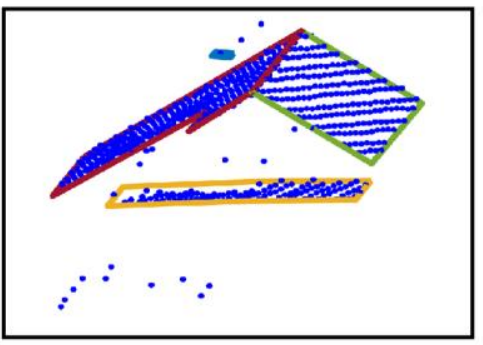

(f)

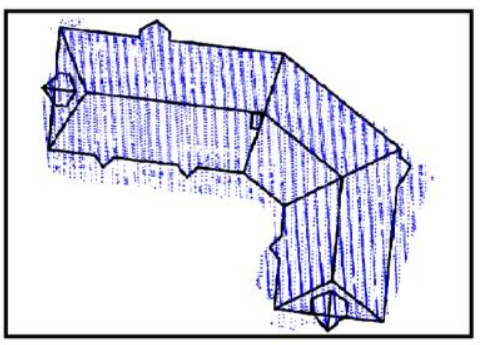

(i)

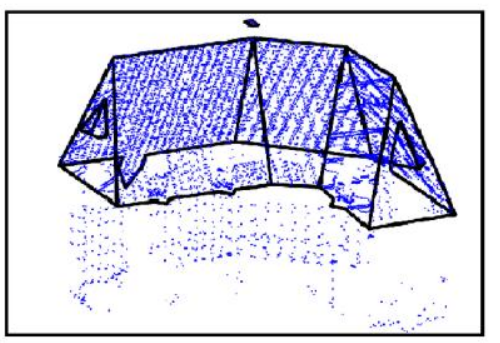

(1)

Figure 3. (a) and (g) Aerial image of Buildings 1 and 2; (b) and (h) 2D reference model; (c) and (i) 2D superimposition of manually extracted point cloud over reference model; (d) and (j) 3D superimposition of manually extracted point cloud over reference model; (e) and (k) 2D superimposition of PCFRM (Point Cloud Fitting Reference Model) over reference model; (f) and (1) 3D superimposition of PCFRM over reference model. 
In order to evaluate the three obtained results, the reference-building model is superimposed over the global point cloud and then whole points located on and inside the building reference model outer boundaries are detected.

According to this definition, a new building point cloud is detected called PCFRM (Point Cloud Fitting Reference Model) (Figure 3 and Table 2). It is noted that the number of points of the PCFRM differs from the number of points of extracted point cloud in the two tested buildings (Figure 3 and Table 2). This difference may be negative (the case of Operator 2 in Building 1), but in the majority of cases it is positive, and it may have a great value (like the case of Operator 3 in Building 1, which is 74.7\%). However, the difference of the number of points between the manually extracted point clouds and the PCFRM may be due to several reasons as follows:

- The reference model does not take into account the spatial decorations, objects and details attached to building facades.

- The horizontal accuracy of point cloud permits some points located nearby the outer building boundary to be outside the building boundary.

- The presence of noisy points attached to building facades due to the presence of vegetation.

- Presence of the confusing points in the neighborhood of building facades.

As the PCFRM is extracted by using the reference model, it can be considered at this stage as a reference point cloud. Consequently, the variation of the number of points between the extracted point clouds (in manual or automatic method) and the PCFRM is considered as an error of building point cloud extraction (Equation 1). Moreover, Equation 2 calculates the percentage of the extraction error.

$$
E_{\mathrm{e}}=N_{\mathrm{pe}}-N_{\mathrm{pp}}
$$




$$
P_{\mathrm{ee}}=\frac{E_{\mathrm{e}}}{N_{\mathrm{pp}}} \times 100
$$

In above equations, $E_{\mathrm{e}}$ is the extraction error, $N_{\mathrm{pe}}$ is the number of points of extracted building cloud, $N_{\mathrm{pp}}$ is the number of points of PCFRM and $P_{\mathrm{ee}}$ is the percentage of the extraction error.

Once the error of building point cloud extraction is calculated, the undesirable point quantity in the building point cloud has to be assessed.

\subsection{Undesirable point quantification}

At this stage, it is important to analyze the building elements presented by the PCFRM. From Figure 3, it is observed that the elements presented by the PCFRM may be categorized into four classes. These classes are the roof points, the facade points, the surrounding terrain points, and the noisy points. However, as the majority of modelling algorithms consider only the roof points during the modelling procedures (Park et al. 2019, Shan et al. 2018 and Tarsha Kurdi et al. 2019), therefore, the last three classes are considered as undesirable points. Therefore, the points of the PCFRM may be classified into two classes, which are roof points and undesirable points. In order to determine the number of points included within each class, the PCFRM of the two selected buildings is manually segmented according to these two classes. The result of this test is presented in Table 2. From Table 2, it can be noted that the majority of PCFRM points belong to the roof class. Moreover, the number of roof points is very close or identical between the manually extracted point cloud and the PCFRM, because the confusing points are actually related to building facades. Nevertheless, the points of the error of building point cloud extraction presented in the last section and the undesirable point class have the same role during the building modelling operation which is the role of undesirable points as explained above. Moreover, they represent to the same building elements (terrain, facades and noise). That 
is why the two-point groups are considered as undesirable point class. Consequently, the extracted building point cloud is composed of two classes which are the roof class and the undesirable point class (Equation 3). That is why the percentage of the non-roof points (undesirable points) in the two tested buildings becomes elevated. Thereafter, Equation 4 can calculate the percentage of undesirable points.

$$
\begin{gathered}
N_{\mathrm{upc}}=N_{\mathrm{pe}}-N_{\mathrm{rp}} \\
P_{\mathrm{upc}}=\frac{N_{\mathrm{upc}}}{N_{\mathrm{rp}}} \times 100
\end{gathered}
$$

In above equations, $N_{\text {upc }}$ is the number of undesirable points, $N_{\text {pe }}$ is the number of points of extracted building cloud, $N_{\mathrm{rp}}$ is the number of points of the roof cloud and $P_{\mathrm{upc}}$ is the percentage of undesirable point class.

Table 2. Error of building point cloud extraction and undesirable points of selected buildings.

\begin{tabular}{|c|c|c|c|c|c|c|c|}
\hline & & $\begin{array}{c}N_{\mathrm{pe}} \\
(\text { Points })\end{array}$ & $\begin{array}{c}E_{\mathrm{e}} \\
(\text { Points })\end{array}$ & $\begin{array}{c}P_{e e} \\
(\%)\end{array}$ & $\begin{array}{c}N_{r p} \\
(\text { points })\end{array}$ & $\begin{array}{c}N_{\text {upc }} \\
(\text { points })\end{array}$ & $\begin{array}{c}P_{\text {upc }} \\
(\%)\end{array}$ \\
\hline \multirow{4}{*}{ Building 1 } & Operator 1 & 711 & 107 & 17.7 & 583 & 128 & 22.0 \\
\cline { 2 - 8 } & Operator 2 & 594 & -10 & -1.7 & 579 & 15 & 2.6 \\
\cline { 2 - 8 } & Operator 3 & 1055 & 451 & 74.7 & 583 & 472 & 81.0 \\
\cline { 2 - 9 } & PCFRM & 604 & -- & -- & 583 & 21 & 3.6 \\
\hline \multirow{3}{*}{ Building 2 } & Operator 1 & 10080 & 938 & 10.3 & 8370 & 1710 & 20.4 \\
\cline { 2 - 8 } & Operator 2 & 10244 & 1102 & 12.1 & 8370 & 1874 & 22.4 \\
\cline { 2 - 8 } & Operator 3 & 12836 & 3694 & 40.4 & 8370 & 4466 & 53.4 \\
\cline { 2 - 8 } & PCFRM & 9142 & -- & -- & 8370 & 772 & 9.2 \\
\hline
\end{tabular}

Once the result of the first experiment is available, the second experiment is achieved by only one operator on three datasets (see Figure 1) for testing the percentage of undesirable points in the building point clouds. Table 3 shows the average number of points of the 
tested buildings in each sample, in addition to the average percentages of undesirable points in building point clouds.

From Tables 2 and 3, it can be observed that the percentages of the undesirable points have sometimes considerable values $(81 \%)$ especially in the case of high buildings (see Toronto sample in Table 3) or in the case of dense vegetation surrounding buildings (see Vaihingen sample in Table 3). Moreover, the percentages of undesirable points are variant according to the operator in the case of manual building extraction. In any way, the density and the homogeneity of facade points play an essential role in the percentages of the undesirable points.

Finally, more investigations are envisaged for analysing the undesirable point class especially in the case high density of facade points.

Table3. Average values of three datasets undesirable point percentages.

\begin{tabular}{|c|c|c|c|}
\hline Dataset & Number of buildings & $N_{\text {pe }}$ (Points) & $P_{\text {upc }}(\%)$ \\
\hline \multicolumn{3}{|c|}{} & \multicolumn{2}{|c|}{ Average values } \\
\hline Toronto & 7 & 36682 & 69.2 \\
\hline Strasbourg & 14 & 2499 & 14.2 \\
\hline Vaihingen & 30 & 3100 & 69.1 \\
\hline
\end{tabular}

At this stage, more investigations are required in order to detect automatically the building roof cloud. Once the undesirable point quantity is assessed, the altimetry comparison of the roof point cloud with the reference model can be achieved.

\subsection{Altimetry comparison}

In the last section, the building points are categorized into two classes, which are undesirable points and roof points. Therefore, the building point cloud in this section refers to the building roof point cloud. In the context of the comparison between the point cloud and the reference model, it may be noted that the geometric nature of the reference model is different from the point cloud. Indeed, a building reference model is a 3D 
polyhedral but the point cloud is a list of 3D points distributed on the building roof surfaces. In order to compare the reference model with the point cloud, the reference model is transformed into a 3D point cloud, and then the two point-clouds are compared. In fact, the adopted method represents exactly the computation of the deviations between the building point cloud and the reference model. This method is called the deviation analysis method (Anil et al. 2013). For calculating the distances of a 3D point to a given plane, the point is projected on the plane and, then, the distance between the point and its projected position is calculated.

First, for a reference model its roof planes are sorted in ascending order of their areas. Then, for each plane, starting from the smallest one because the roof detail planes may be included within the principal roof planes, we find the points within the plane. These points $P=(X, Y, Z)$ are marked as "used" so that they are not used for another plane. By using the plane equation, the projected position $P_{p}=\left(X, Y, Z_{p}\right)$ is calculated. Thus, we get a calculated reference point cloud for the reference model. Finally, the difference in $Z$ value $\left(Z-Z_{p}\right)$ is obtained for the reference model. These differences represent the deviations between the roof point cloud and the reference model. In order to analyze them, the $\left(Z-Z_{p}\right)$ values are clipped to be between $-1 \mathrm{~m}$ to $+1 \mathrm{~m}$ because the extreme values of $(\mathrm{Z}-\mathrm{Zp})$ represent obstacles in the visualisation and graphic illustrations. In this context, the histograms of the deviations between the reference and the Lidar point clouds is shown in Figure 4. From these histograms, it can be noted that the roof point cloud can be classified into two classes, which are the class of roof points and the class of noisy points.

Concerning the class of roof points, in the standard case, the roof points have to be distributed statistically in an equal way around the two sides of roof planes according to 
the least squares theory. Unfortunately, that is not the case in the two tested buildings. From Figure 4a, it can be seen in the histogram of Building 1 that the number of points situated over the reference planes is significantly greater than that situated under the reference planes. By contrast, from Figure $4 b$, it can be seen that the majority of points of the second building are situated under the reference planes. This observation shows that the reference roof planes do not represent accurately the constructed planes. In fact, in the reference models, only the boundary corner point coordinates define each roof plane equation. In the original building, the roof surfaces do not represent perfect planes. Furthermore, the roof geometric form and thickness of the texture are not considered in the construction of the reference models. Otherwise, in Lidar data, a great number of 3D points present the roof surface. However, in order to be fair, the coordinates $X$ and $Y$ of reference plane boundary corners are sufficiently accurate because for calculating the best mean roof planes, only the $Z$ coordinates of the reference roof plane boundary corners have to be adjusted.

From Figure 4, it can be noted that the majority of roof points have deviations less than $0.35 \mathrm{~m}$. In fact, this value is related to the altimetry accuracy of the point cloud, the faithfulness level of roof plane surfaces toward the perfect plane form and the roof texture quality. Therefore, all roof points having deviations belonging to the interval $[-0.35 \mathrm{~m}$, $+0.35 \mathrm{~m}]$ are considered as part of the class of roof points, otherwise they are considered as noisy class. Table 4 exposes the percentages of points belonging to the last two classes of the two selected buildings. Moreover, the red ovals in Figure 4 refer to the noisy point class. It can be noted from Table 4 that the percentage of noisy points in Building 2 is elevated (noisy points $=5.6 \%$ ) in comparison to Building 1 (noisy points $=1.2 \%$ ). In order to explain why this percentage is high, the noisy points are superimposed on the reference model (Figure 5b). The comparison between the building aerial image (Figure 5a) and 
the image of superimposition of noisy points on the reference model (Figure 5b) shows that the majority of the noisy points present roof details that are not considered in the reference model (see red circles in Figure 5b).

Table 4. Roof point class and noise class in roof point cloud.

\begin{tabular}{|c|c|c|c|}
\hline & $\begin{array}{c}\text { Roof point class, } \\
\text { deviations inside } \\
{[-0.35,+0.35](\%)}\end{array}$ & $\begin{array}{c}\text { Noisy class, } \\
\text { deviations outside } \\
{[-0.35,+0.35](\%)}\end{array}$ & $\begin{array}{c}\text { Number of } \\
\text { points of the } \\
\text { roof }\end{array}$ \\
\hline Building1 & 98.8 & 1.2 & 583 \\
\hline Building2 & 94.4 & 5.6 & 8370 \\
\hline
\end{tabular}

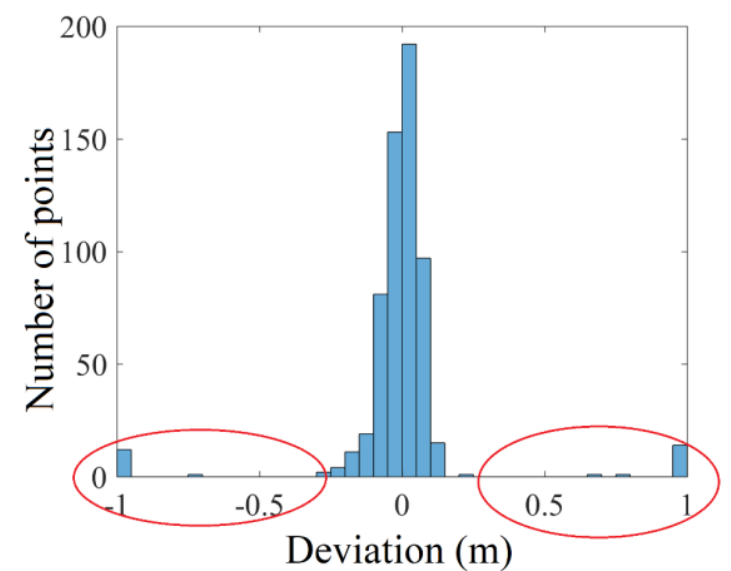

Deviation (m)

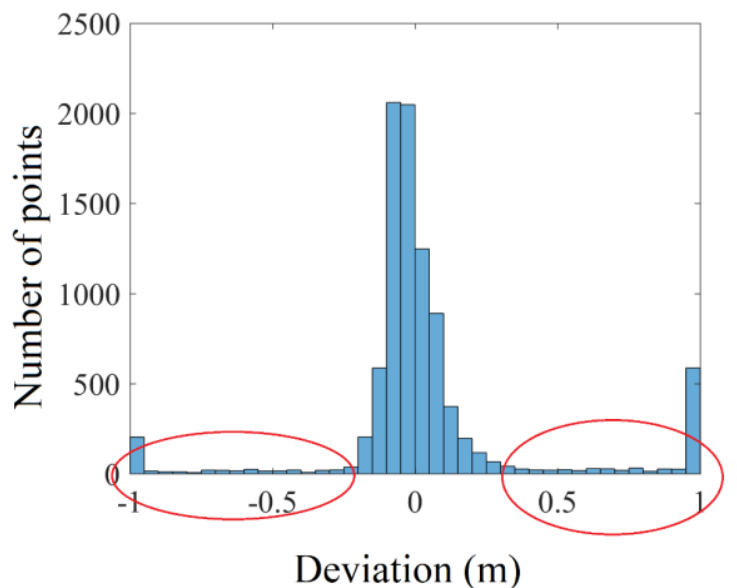

Deviation (m)

Figure 4. Histograms of deviations between roof point cloud and reference model; (a) Building 1; (b) Building 2; Red ovals present the noisy points.

Consequently, if all roof details are considered in the reference model of Building 2, the percentage of noisy points will be $1.49 \%$ which represents a reasonable value (between 1 and 2\%), the same as the case of Building 1 . Figure $5 \mathrm{c}$ illustrates the reference model of Building 2 after considering all roof details. At this stage, more investigations are required for analyzing the sources of the noisy point class in the roof point cloud. 


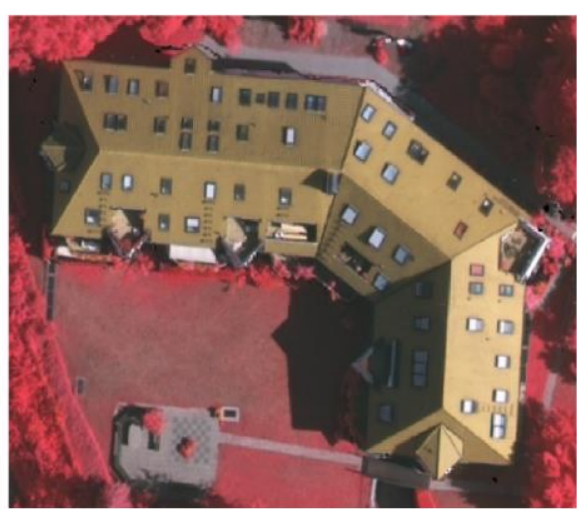

(a)

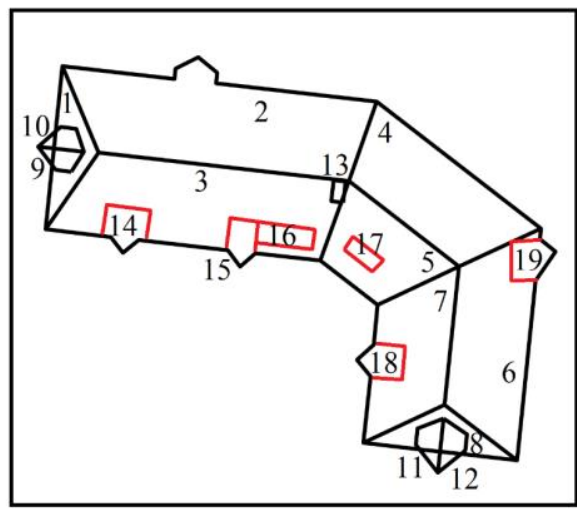

(c)
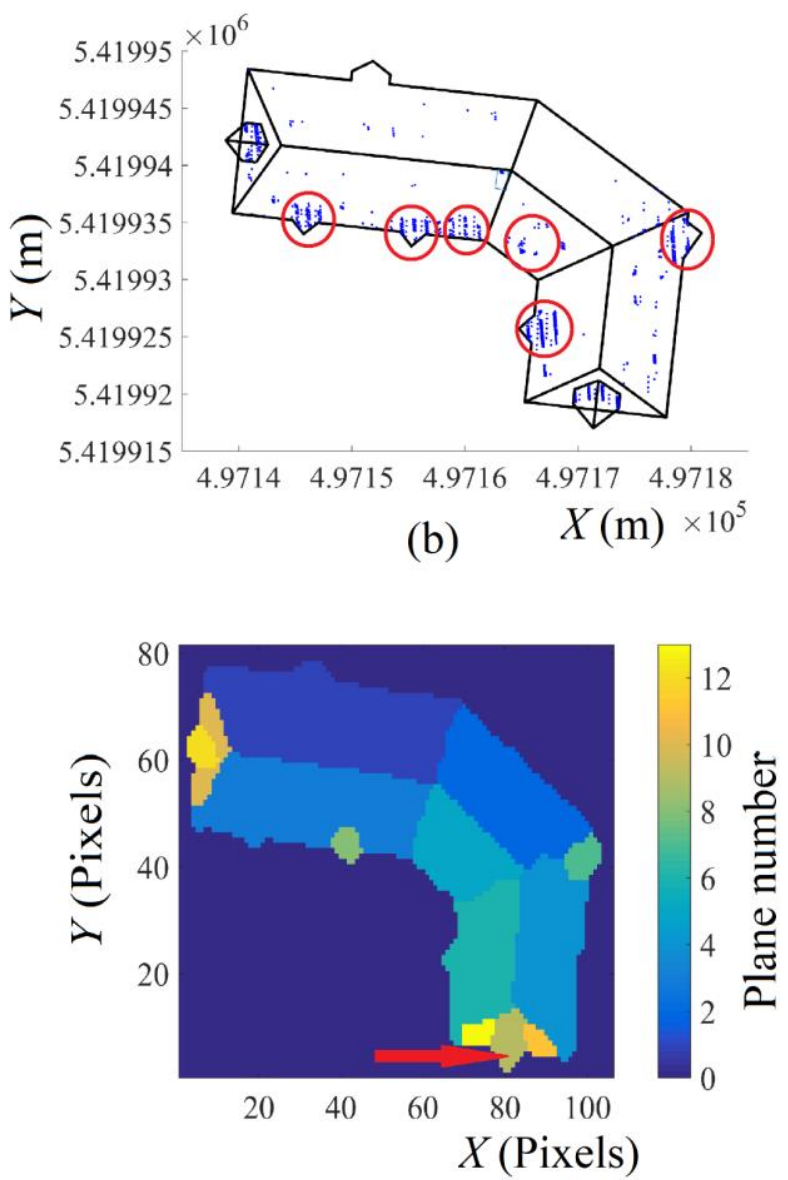

(d)

Figure 5. (a) Aerial image; (b) Superimposition of noisy points on reference model; Red circles: noisy points presenting roof details that are not considered in reference model; (c) Complete reference model; Red polygons presents missing details in reference model; (d) Second automatic segmentation trail result; Red arrow points to the merged details.

\subsection{Segmentability of roof point cloud}

A building reference model is composed of a list of plane segments. These segments represent the roof surfaces. The roof segmentability is the possibility for roof segmentation according to its composed surfaces. In the context of $3 \mathrm{D}$ building modelling, the more delicate step in data-driven approaches is the segmentation of a building roof point cloud (Park et al. 2019). Indeed, any misdetection of the roof planes will generate undesirable differences in the 3D building model. In order to segment a roof 
point cloud, two methods are envisaged, automatic segmentation and manual segmentation. The automatic building roof segmentation topic is covered widely in the literature and many approaches have been suggested to achieve this goal (Tarsha Kurdi and Awrangjeb 2020). Despite manual segmentation being very slow in comparison to automatic segmentation, it is still supposed to be more accurate, especially if an expert in the Lidar data processing field generates it. Indeed, in the manual method, the segmentation is achieved point per point and the operator may have extra information about the scanned building such as aerial photos, building plan and/or other collected information, in addition to supplementary informatics navigation and visualization tools. That is why Shan et al. (2018) derive the reference model by manual segmentation of the building point cloud.

This section discusses the segmentability of a roof point cloud. Two questions arise; firstly, do all building point clouds have the same segmentability level? Secondly, is the mis-segmentation due to the point cloud quality or due to the segmentation algorithm?

\subsubsection{Selected building samples}

In order to answer these questions, three building point clouds are selected of different typology and different point density. The first two buildings belong to Vaihingen point cloud where the point density equals to $4-6.7$ points $/ \mathrm{m}^{2}$ whereas the third building is taken from Strasbourg point cloud with a low point density of 1.3 points $/ \mathrm{m}^{2}$ (see Table 1). In this experiment, the cloud points cover only the roofs meaning there are no extracted erroneous points and points on building facades.

In this context, the building point cloud is segmented into three classes, which are principal plane points, detail points and noisy points (Table 5). The principal planes cover most of the roof area and the area of each one is large in regard to the roof detail areas. 
The detail planes include windows, chimneys, antennas, tanks, decorative features, or objects that may be found on the roof. The area threshold that separates between the two plane classes is not always clear, because some buildings may have a high gradation in roof plane areas, which makes it difficult to distinguish the principal planes from detail planes. At last, despite the elimination of both extracted erroneous points and facade points, noisy points remain as seen in Section 4.3.

Concerning the architectural complexity of the selected buildings, Building 1 (Figure 3) is very simple and has only three principal roof planes and one roof detail. Building 2 (Figure 3) is more complex than the first one and its roof contains eight principal planes in addition to 11 details (Figure 5c). Building 3 (Figure 7) represents a high architectural complexity in addition to weak point density that is why its roof point cloud contains only 1488 points despite its great area (more than $1400 \mathrm{~m}^{2}$ ). Its roof is composed of 111 principal and detail planes with high gradation in roof plane areas (see Table 5).

\subsubsection{Experimental setup}

Three skilled operators segment each one of the selected buildings manually. Both the aerial images and the building point clouds of the tested buildings are provided to every operator. Moreover, the tested building point clouds are segmented automatically three consecutive times by using the extended RANSAC algorithm suggested by Tarsha Kurdi et al. (2008). More details about this algorithm are exposed in the next section. In this context, the order of detected planes by the extended RANSAC algorithm is not identical in consecutive trials because it depends on the probability law for detecting the best plane (see next section). Finally, the selected buildings are segmented by superimposing the reference model over the point cloud, which means they are segmented according to the 
reference models. The last segmentation result is named SRM (Segmentation by Reference Model).

\subsubsection{Experimental results}

In order to analyze these results, two viewpoints are employed. The first one considers only the number of roof plane segments whereas the second viewpoint takes into account the number of points in the roof plane segments. Tables 5, 6 and 7 in addition to Figures 6 and 7 show the experimental results of Section 4.4 .

Table 5. Segmentation results of three selected buildings by considering number of roof segments.

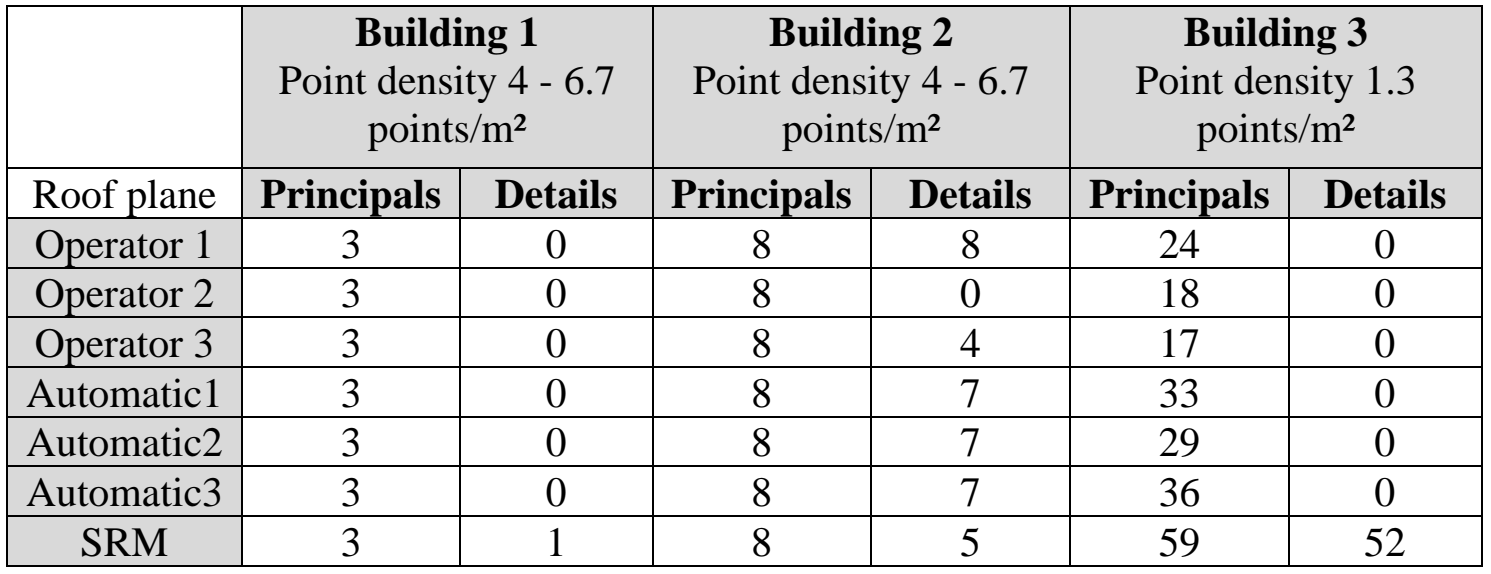

Table 6. Trial results of roof cloud segmentation of Building 1.

\begin{tabular}{|c|c|c|c|c|c|c|}
\hline & $\begin{array}{l}\text { Plane1 } \\
\text { (points) }\end{array}$ & $\begin{array}{l}\text { Plane } 2 \\
\text { (points) }\end{array}$ & $\begin{array}{l}\text { Plane } 3 \\
\text { (points) }\end{array}$ & $\begin{array}{l}\text { Plane } 4 \\
\text { (points) }\end{array}$ & $\begin{array}{l}\text { Noise } \\
\text { (points) }\end{array}$ & $\begin{array}{l}\text { Total } \\
\text { (points) }\end{array}$ \\
\hline Operator 1 & 96 & 286 & 198 & 0 & 3 & \multirow{7}{*}{583} \\
\hline Operator 2 & 82 & 264 & 195 & 0 & 42 & \\
\hline Operator 3 & 69 & 254 & 198 & 0 & 62 & \\
\hline SRM & 98 & 275 & 193 & 2 & 15 & \\
\hline Automatic 1 & 93 & 259 & 222 & 0 & 9 & \\
\hline Automatic 2 & 93 & 260 & 221 & 0 & 9 & \\
\hline Automatic 3 & 93 & 262 & 218 & 0 & 10 & \\
\hline
\end{tabular}


Table 7. Segmentation trial results of the principal roof planes in Building 2.

\begin{tabular}{|c|c|c|c|c|c|c|c|c|}
\hline & $\begin{array}{c}\text { Plane1 } \\
\text { (points) }\end{array}$ & $\begin{array}{c}\text { Plane 2 } \\
\text { (points) }\end{array}$ & $\begin{array}{l}\text { Plane 3 } \\
\text { (points) }\end{array}$ & $\begin{array}{c}\text { Plane 4 } \\
\text { (points) }\end{array}$ & $\begin{array}{c}\text { Plane5 } \\
\text { (points) }\end{array}$ & $\begin{array}{c}\text { Plane 6 } \\
\text { (points) }\end{array}$ & $\begin{array}{c}\text { Plane7 } \\
\text { (points) }\end{array}$ & $\begin{array}{c}\text { Plane 8 } \\
\text { (points) }\end{array}$ \\
\hline Operator 1 & 338 & 1976 & 1416 & 1081 & 527 & 1263 & 1045 & 220 \\
\hline Operator 2 & 325 & 1985 & 1264 & 1098 & 580 & 1023 & 1025 & 267 \\
\hline Operator 3 & 238 & 2035 & 1459 & 1138 & 546 & 1246 & 1107 & 136 \\
\hline SRM & 149 & 2059 & 1302 & 1188 & 571 & 1181 & 1089 & 184 \\
\hline Automatic 1 & 162 & 2096 & 1371 & 1198 & 573 & 1206 & 1063 & 189 \\
\hline Automatic 2 & 183 & 2065 & 1375 & 1181 & 547 & 1224 & 1093 & 188 \\
\hline Automatic 3 & 338 & 1976 & 1416 & 1081 & 527 & 1263 & 1045 & 220 \\
\hline
\end{tabular}
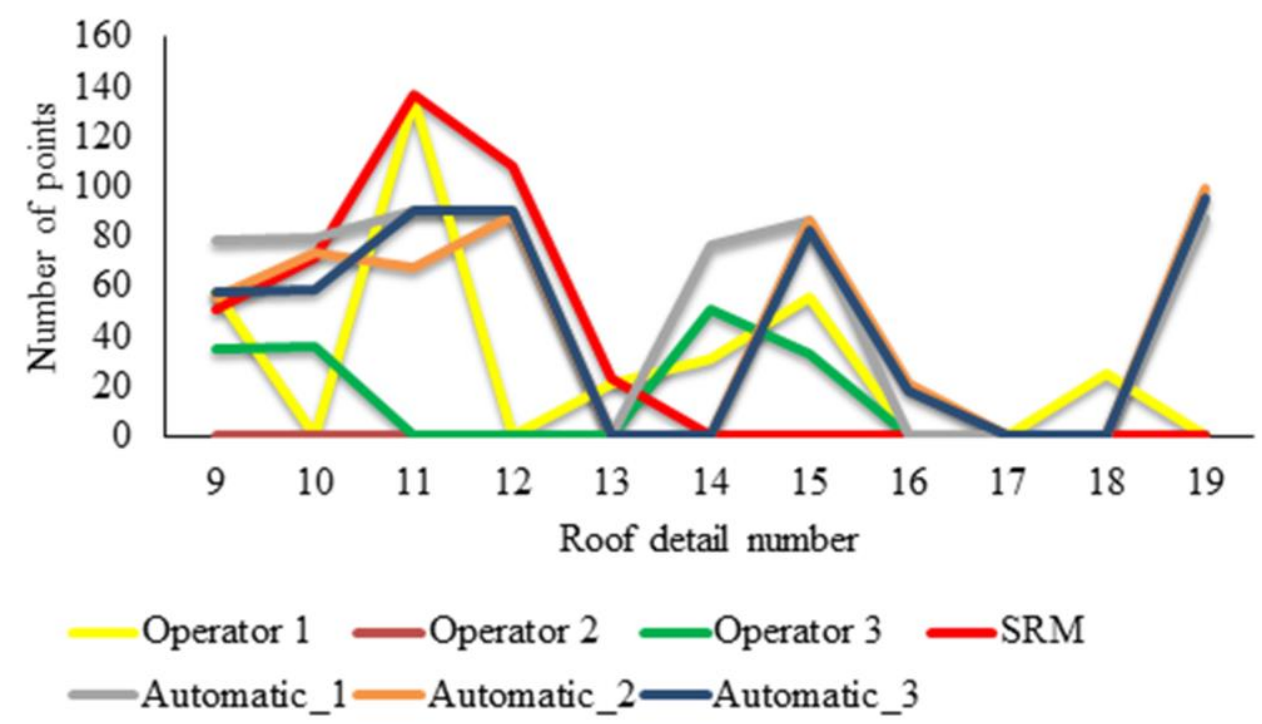

Figure 6. Number of points of the detail roof planes in Building 2.

\section{(a) Comparison of number of roof segments}

In Table 5, the roof planes are categorized according to their areas into two classes: the roof details and the principal roof planes. It can be noted that all principal planes are detected in Buildings 1 and 2. In Building 3, regarding the high gradation in roof plane areas in addition to the low point density, only planes having considerable areas are detected correctly. Moreover, the roof planes having small areas are wrongly segmented. That is why the number of detected principal planes is between 17 planes and 36 planes in all tests while the reference model contains 59 principal planes. 
Concerning the roof details, the single detail in Building 1 is never detected because only two points represent it (Table 6). In Building 2, the reference model considers only 5 out of 11 roof details (see Figure 5c). Figure 6 shows that except for Plane 17, each one of the ten roof details are detected at least one time during the achieved trials. In the automatic and manual trials, sometimes the neighboring details are merged together (see red arrow in Figure 5d) and sometimes they are detected separately. However, all roof details are never detected at once in Building 2, the maximum number of detected details is 6 by Operator 1, and there always remain missed details. Finally, the roof details in Building 3 are never detected regarding to the weak point density (Figure 7 and Table 5). From Table 5, the effectiveness of automatic segmentation is often equal to or better than the manual segmentation because the number of detected planes in automatic segmentation are almost equal to (in case of Building 1) or greater than the case of manual segmentation. In conclusion, the segmentability of the three tested building point clouds is different regarding both the stability of the segmentation results, and the fulfilment level of segmentation results toward the original buildings. The order of the tested buildings regarding the segmentability and starting from the best case is Building 1, Building 2 and, then, Building 3.

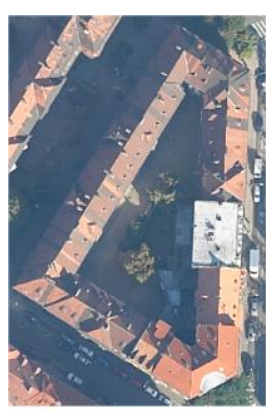

(a)

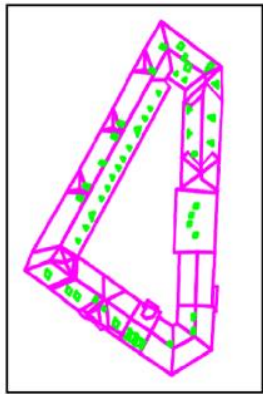

(b)

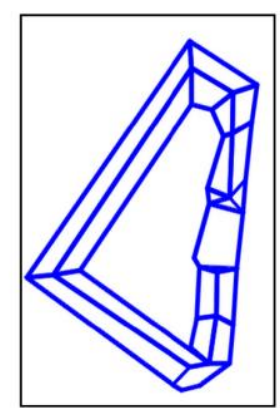

(c)

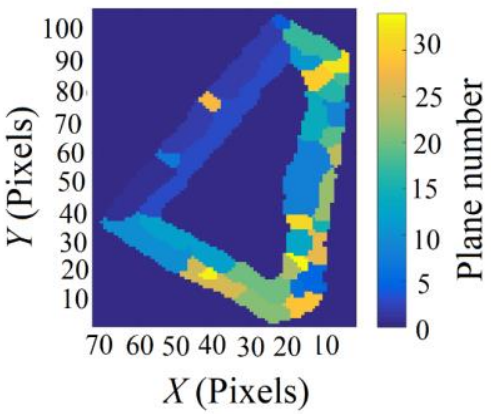

(d)

Figure 7. For Building 3: (a) Aerial image; (b) Reference model; Roof details are in green color; (c) Manual segmentation result by Operator 2; (d) First trial of automatic segmentation result. 


\section{(b) Comparison of number of points of roof segments}

Table 6 exposes the segmentation trial results of Building 1 where the number of points of each roof segment, in addition to the number of noisy points, are presented. In each manual trial, the number of points of Plane 2 is different. The same remark is also observed for Plane 3. In order to explain this difference, it is sufficient to note that the mutual relationship between these two neighboring planes is the intersection. Thereby, the pertinence of the points around the intersection line is sometimes fuzzy for the operators. That is why the number of plane points is different in each trial. In the case of Plane 1, the three operators disagree about all points belonging to this plane, because there is a remarkable irregularity of point distribution in the altimetry and the horizontal levels.

In the same context, in the automatic trials, the number of points of Plane 3 is greater than those in the manual trials. Contrariwise, the number of points of Plane 2 is less than those of the manual trials. In order to understand the reason for this difference, it is important to note that the plane definition used in the employed automatic segmentation algorithm (using the extended RANSAC) is completely different from that of the traditional geometry. On the one hand, a plane in the traditional geometry is a surface containing the straight line in all directions. On the other hand, in the extended RANSAC algorithm, one plane is a thickness of points ranging between two parallel planes (Tarsha Kurdi et al. 2008). The use of this plane definition generates an error $\varepsilon$ if the detected plane intersects with one of its adjacent planes (see Figure 8) which is the reason for the jump in the number of points between automatic and manual segmentations in the case of Planes 2 and 3.

Moreover, the number of points of Plane 2 in the automatic trials is different in each result and the same thing for Plane 3. In fact, the employed extended RANSAC algorithm 
depends on the probability law for detecting the best plane by $N$ random selections of three points from building cloud. That leads to a tiny difference in the detected plane equation in each trial result according to the selected three points. Consequently, the value of the error $\varepsilon$ in the case of intersecting neighboring planes will be slightly different from one trial to other. This remark leads also to understand why the number of points of Plane 1 in automatic trials is equal in the three results. However, the difference of number of points between the automatic trial results in this case is negligible and the used segmentation algorithm can be considered stable. Furthermore, the error $\varepsilon$ mentioned above may be cancelled by improving the segmentation algorithm.

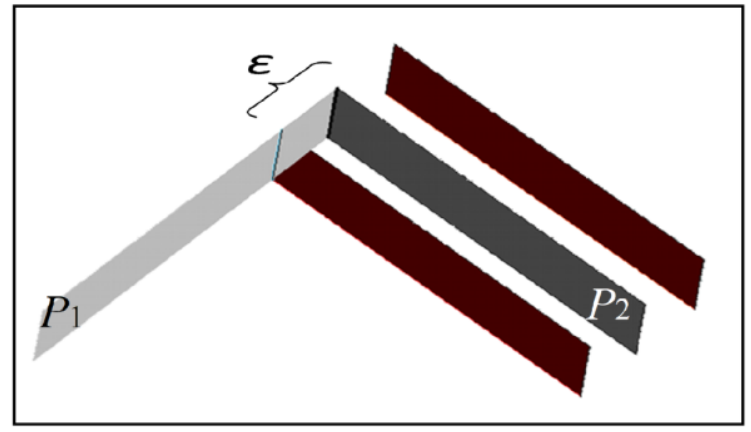

(a)

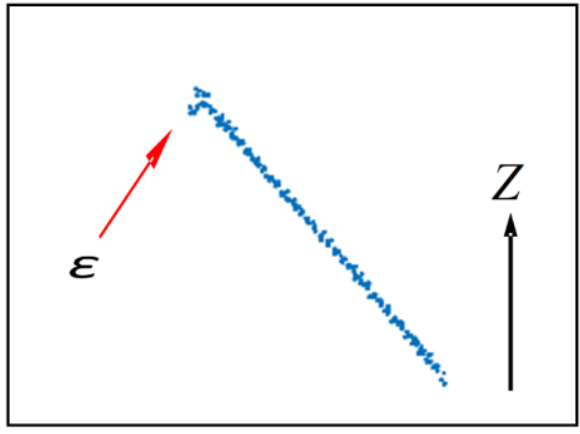

(b)

Figure 8. (a) Error $\varepsilon$ of plane detection in the case of intersection mutual relationship between adjacent planes. (b) Error $\varepsilon$ of automatic detection of Plane 3 in Building 1.

Concerning the noisy points, these points are located within the outer building boundary polygon and are not considered to belong to any one of the roof planes. In the manual segmentation trials, one operator has several instructions for considering whether one point is a noisy point or not. Some of these instructions are the distance between the point and the nearest plane, and the respecting of the regularity of the selected plane boundary. 
As every operator has its own viewpoint, the differences between the numbers of noisy points in the manual trials are understandable.

Table 7 illustrates the segmentation trial results on the principal roof planes in Building 2 , where the number of points of each principal roof segment is presented. Figure 6 illustrates graphically the number of points of the detail roof planes in Building 2. In this figure, if the detail number of points equals to zero that means this detail is missed.

As shown in Figure 5c, the roof of Building 2 contains eight principal planes in addition to 11 details. Only five details are considered in the reference model and the other ones are missed. Moreover, in each segmentation trial, some details are detected and the others are missed (Figure 6). As the paper topic focuses on the comparison of the building point cloud to the reference model, the case of an uncompleted reference model is expected in regard to the multiple constructing methods of reference model (see Section 2.1). Consequently, it is necessary to keep the reference model of Building 2 unchanged. In fact, in the manual trials, if one roof detail is missed, that will not affect the result of detection of the other roof planes. Contrariwise, in the automatic segmentation, when one roof detail is missed, the points covering this detail will be added to the principal plane containing this detail. Consequently, these points will represent undesirable noise, which will deform the equation of the principal plane containing this detail. Moreover, if one principal plane equation is deformed, and this plane has neighboring planes with an intersection mutual relationship, the boundaries between this plane and its neighbors will be deformed and perhaps shifted. Therefore, in Table 7, considerable differences may be observed in the number of points of each plane between the several segmentation trials.

To conclude, any plane not presented in the reference model and detected by segmentation procedures provides a false alarm about the accuracy. Contrariwise, any 
plane of the reference model not detected by the involved segmentation procedure will decrease the segmentation accuracy and may generate differences in the plane boundaries. According to Tarsha Kurdi and Awrangjeb (2020), the missing planes can be localized by analyzing the error map matrix. This matrix can also help to control the reference model before using it in testing the accuracy of the calculated model. Finally, it can be concluded that the segmentability of the building point cloud depends on the point cloud characteristics such as the point density and the accuracy, the roof architectural complexity, and the competence of the segmentation algorithm.

\subsection{Per plane comparison}

When the roof point cloud is superimposed on the reference model, the point cloud can be segmented according to the planes composing the reference model. The objective of this section is to compare each plane in the reference model with its corresponding list of points. This comparison will take into account two viewpoints, which are the plane area and the point distribution within the plane area.

In order to carry out these comparisons, the three principal planes of Building 1 (Figure 3a) are considered where the fourth plane represents a roof detail and containing only two points that is why it is not considered (see Table 6). Figure 9 shows the superimposition of the plane boundaries extracted manually (green color), the reference plane boundaries (red color) and the plane Lidar points (blue circles). 


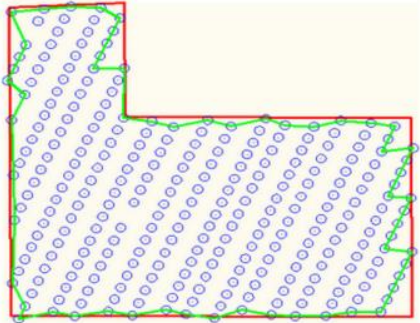

(b)

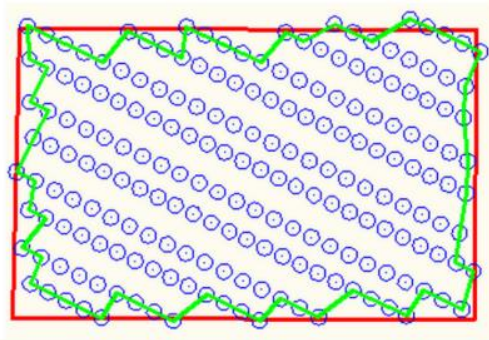

(c)

Figure 9. Comparison between plane boundaries extracted manually and reference model in Building 1; (a) Plane 1; (b) Plane 2; (c) Plane 3; Plane numeration is taken from Figure 1b; Red: reference plane boundaries; Green: manually extracted plane boundary; Blue circles: Lidar points.

\subsubsection{Comparison of plane areas}

Table 8 illustrates the comparison between the reference areas and the manually detected areas that envelope the plane Lidar points. It can be noted that one manually detected area is always smaller than the reference area, because the Lidar points do not entirely cover the plane area. At this stage, a new factor is defined named covering ratio. Its value is calculated by dividing the point envelop area on the reference plane area (Table 8). The covering ratio factor represents an indice of the percentage of plane area covered by Lidar points without considering the spaces between them. This factor value is related to the point density. Indeed, when the point density is more elevated, then the number of points covering one roof plane also becomes greater. Consequently, the spaces between the reference plane and the polygon enveloping the Lidar points will become smaller. Moreover, if there is an obstacle nearby the building such as a high tree or a high building, and this obstacle partially prevents scanning one roof plane, the quantity of points covering this roof plane in this case will decrease as the Plane 1 in Figure 9. This error is not only related to the presence of obstacle nearby the building, but it is also related to the location of the building among the scan strip. If the building is located far from the 
strip center, then this error value will be greater than the case of the same building located nearby the strip center. However, when the covering ratio value is near to $100 \%$ that means the plane is perfectly covered by Lidar points. Contrariwise, if its value is vastly different from $100 \%$, that means the Lidar points poorly cover the plane. In this case, the plane boundary model may be deformed.

In this stage, it is beneficial to estimate the error of the plane areas. For this purpose, the confusion matrix is employed for calculating the correctness and the completeness accuracy indices (Shan et al. 2018). In this context, three elements from the confusion matrix will be employed which are:

- TP (True Positive): the common area of both reference and detected plane

- FN (False Negative): the area of the reference not found in the detected plane

- FP (False Positive): the area of the detected plane not found in the reference

Table 8 represents the TP, FN and FP values, in addition to the Corr (Correctness) and the Com (Completeness) values. It can be observed that all FP values are equal to zero and the Corr values are equal to100 \%. Indeed, the segmentation of roof point cloud is carried out by superimposing the reference model on the roof point cloud and all roof points are located within the plane boundaries.

Table 8. Values of areas, confusion matrix elements, modal density and true density for Building 1 roof planes; RA: Reference Area; EA: Enveloping Area; CR: Covering Ratio; NP: Number of Points; MD: Modal Density; TD: True density.

\begin{tabular}{|l|l|l|l|l|l|l|l|l|l|l|l|}
\hline & $\begin{array}{l}\text { RA } \\
\left(\mathrm{m}^{2}\right)\end{array}$ & $\begin{array}{l}\text { EA } \\
\left(\mathrm{m}^{2}\right)\end{array}$ & $\begin{array}{l}\text { CR } \\
\%\end{array}$ & $\begin{array}{l}\text { TP } \\
\left(\mathrm{m}^{2}\right)\end{array}$ & $\begin{array}{l}\text { FP } \\
\left(\mathrm{m}^{2}\right)\end{array}$ & $\begin{array}{l}\text { FN } \\
\left(\mathrm{m}^{2}\right)\end{array}$ & $\begin{array}{l}\text { Corr } \\
\%\end{array}$ & $\begin{array}{l}\text { Com } \\
\%\end{array}$ & $\begin{array}{l}\text { NP } \\
(\text { Points })\end{array}$ & $\begin{array}{l}\text { MD } \\
(\text { Points } \\
\left./ \mathrm{m}^{2}\right)\end{array}$ & $\begin{array}{l}\text { TD } \\
(\text { Points } \\
\left./ \mathrm{m}^{2}\right)\end{array}$ \\
\hline Plane 1 & 40,8 & 23.3 & 57.1 & 23.3 & 0 & 17.5 & 100 & 57.1 & 98 & 2.4 & 4.2 \\
\hline Plane 2 & 89.49 & 81.76 & 91.4 & 81.76 & 0 & 7.73 & 100 & 91.4 & 275 & 3.1 & 3.4 \\
\hline Plane 3 & 67.15 & 57.84 & 86.1 & 57.84 & 0 & 9.31 & 100 & 86.1 & 193 & 2.9 & 3.3 \\
\hline
\end{tabular}


Moreover, both the completeness factor and the covering ratio have identical values, which express the percentage of plane area covered by points without considering the spaces between points.

\subsubsection{Point distribution}

In fact, the form of laser footprint within the roof plane area is related to Lidar system quality (Wehr and Lohr 1999). From Figure 9, it can be noted that the Lidar points are distributed in the form of pair of lines recurring consecutively within the roof plane areas. These pairs represent the scan lines. The distance between the lines of the same pair is smaller than the distance between two consecutive pairs. Moreover, there are slight variations in the distance values between each point and its neighboring ones, due to the plane texture, the laser beam scan angle value in addition to the Lidar system calibration errors. If the scan lines are parallel to one of the principal plane axes, in this case, the cover ratio takes a minimal value and one of the plane dimensions length will be deformed.

At this stage, it is important to verify the value of point density. For this purpose, three kinds of point density can be distinguished which are the theoretical density, the modal density and the true density. The theoretical density (Table 1) is normally provided by the Lidar providing company where it is calculated according to the scan parameters by supposing that the scanned area is flat and horizontal and all scan conditions are perfect. The modal density is calculated by dividing the plane number of points on its area. This density value considers that the points are distributed on all plane surfaces regardless of the covering ratio, whereas the true density is calculated by dividing the plane number of points by the area of the point enveloping polygon. 
Table 8 compares the modal and the true point density of the three principal planes of Building 1. It can be observed that the true density value is always greater than the modal one, and it is close to the theoretical density value. Unfortunately, the values of the true density in Planes 2 and 3 are still smaller that the theoretical one. In fact, the true density value is related to the orientation angles of the roof plane and the building location in the scan strip. Moreover, the scan strip contains two different theoretical density values according to the overlapping and the non-overlapping areas. That is why the true density value is different from one roof plane to other. However, both the covering ratio and true point density directly impact the calculated 3D building model quality because some modelling algorithm parameters are a function of point density such as the minimum and the maximum of roof plane areas.

\section{Analysis, perspective and conclusion}

This paper has presented the investigation regarding the understanding of the structure of building Lidar point cloud in order to determine the fidelity level of the cloud toward the original building. The existence of high percentage of undesirable points (between $15 \%$ and $70 \%$ ). in the building cloud presents an obstacle to any involved roof segmentation procedures when the segmentation algorithm considers only the roof points. In this context, it is advised to develop a new algorithm that classifies the building point cloud into two classes: the roof class and the undesirable point class (i.e., non-roof class). Despite the non-roof class being considered as undesirable points, it can be sometimes useful in the case of low covering factor value for modelling the outer plane boundaries. Moreover, in the case of high point density in the facade class, the facade points may be extracted and modelled from the non-roof class.

The advantage of Lidar data in regard to the other acquisition data technologies is that the roof plane equations can be calculated precisely thanks to the presence of great data 
quantity covering the majority of roof surfaces (between 1.3 and 6.7 points $/ \mathrm{m}^{2}$ ). Contrariwise, the precise calculation of planer coordinates $X$ and $Y$ of the inner roof plane boundary corners sometimes presents a challenge due to the roof segmentation errors and the covering ratio (between $57.1 \%$ and $91.4 \%$ ).

According to the point cloud characteristics, the roof architectural complexity, and the competence of the segmentation algorithm, the segmentability of a building point cloud varies from one building cloud to another. Moreover, the segmentability may be variant in the same building from one building part to another (Figure 7). Therefore, it is necessary to employ a supplementary algorithm that can automatically estimate the success level of roof segmentation such as the algorithm suggested by Tarsha Kurdi and Awrangjeb (2020). This algorithm may help to localize the cases of segmentation failures and consequently to find suitable solutions.

The detection and the modelling of most roof details in Lidar data present sometimes a true challenge. Therefore, it is unavoidable, in the case of a successful segmentation, to verify automatically if all roof planes are detected or not. For this purpose, Tarsha Kurdi and Awrangjeb (2020) suggest a new algorithm that can achieve this goal by analysing the error map matrix.

Concerning the point density value, there are three different types of density envisaged: the theoretical density (ThD), the modal density (MD) and the true density (TD) (Section 4.5 where $\mathrm{MD}<\mathrm{TD} \leq \mathrm{ThD}$ ). In this context, it is important to calculate the plane true density values in the course of building modelling algorithm, especially when raster operations are employed. Indeed, some modelling algorithm parameters are a function of point density such as the minimum and the maximum of roof plane areas. Consequently, the precise estimation of point density leads to an appropriate estimation of these algorithm parameters. 
To conclude, in the context of modelling accuracy estimation, the direct comparison of constructed building model with the reference one will neglect the advantages and the disadvantages of building point cloud. Moreover, this comparison will link directly between all obtained differences and the modelling algorithm, which is not always true because a considerable part of building model differences is related to the building point cloud structure. In order to understand globally the building model differences sources and the relationship between the reference model, the point cloud and the constructed building model, future research will focus on the analysis of the relationships between the point cloud and the constructed model in addition to the relationship between the reference model and the constructed one.

Acknowledgement: The Vaihingen data set was provided by the German Society for Photogrammetry, Remote Sensing and Geoinformation (DGPF) (Cramer, 2010): http://www.ifp.uni-stuttgart.de/dgpf/DKEP-Allg.html.

\section{References}

Akca, D., Freeman, M., Sargent, I., and Gruen, A. 2010. "Quality assessment of 3D building data." The Photogrammetric Record, 25(132), pp.339-355.

Anil, E.B., Tang, P., Akinci, B., and Huber, D. 2013. "Deviation analysis method for the assessment of the quality of the as-is Building Information Models generated from point cloud data." Automation in Construction, Volume 35, pp. 507-516, http://dx.doi.org/10.1016/j.autcon.2013.06.003.

Awrangjeb, M., Gilani, S.A.N., and Siddiqui, F.U. 2018. "An effective data-driven method for 3D building roof reconstruction and robust change detection." Remote Sens. 2018, 10, 1512; DOI: 10.3390/rs10101512.

Bossler, J.D., Campbell, J.B., Mcmaster, R.B., and Rizos, C. 2010. Manual of geospatial science and technology. Boca Raton, CRC Press, https://doi.org/10.1201/9781420087345.

Cheng, L., Zhang, W., Zhong, L., Du, P., and Li, M. 2015. "Framework for evaluating visual and geometric quality of three-dimensional models." IEEE journal of selected topics in 
applied earth observations and remote sensing, vol. 8, no. 3, March 2015, DIO:

10.1109/JSTARS.2014.2370753.

Cramer, M. 2010. "The DGPF test on digital aerial camera evaluation - overview and test design.” Photogrammetrie - Fernerkundung - Geoinformation 2(2010):73-82.

Demir, N. 2018. "Automated detection of 3D roof planes from Lidar data." Journal of the Indian Society of Remote Sensing. August, 46(8): pp.1265-1272. https://doi.org/10.1007/s12524-018-0802-2.

Dorninger, P., and Pfeifer, N. 2008. "A comprehensive automated 3D approach for building extraction, reconstruction, and regularization from airborne laser scanning point clouds." Sensors, 8(11), pp.7323-7343.

Edirisinghe, R., and London, K. 2015. "Comparative analysis of international and national level BIM standardization efforts and BIM adoption." Proceedings of the 32nd Int Conference CIB W78, Eindhoven, The Netherlands, 2015, pp. 149-158.

Elberink, S.O., and Vosselman, G. 2011. "Quality analysis on 3D building models reconstructed from airborne laser scanning data." ISPRS Journal of Photogrammetry and Remote Sensing, 66(2), pp.157-165. http://doi.org/10.1016/j.isprsjprs.2010.09.009.

Gülch, E., Kaartinen, H., and Hyyppä, J. 2018. "Quality of buildings extracted from airborne laser scanning data-results of an empirical investigation on 3D building reconstruction." In: Shan, J. and Toth, C.K. 2ndEd, Topographic Laser Ranging and Scanning: Principles and Processing. CRC Press: Boca Raton, Taylor \& Francis Group, FL, pp. 587-621.

Guptill, S. C., and Morrison, J. L. 1995. Elements of spatial quality. Elsevier Science, Kidlington, Tarrytown, Tokyo, ISBN 0080424325 hardcover.

Habib, A. 2018. "Accuracy, quality, assurance and quality control of light detection and ranging mapping." In: Shan, J. and Toth, C.K. 2ndEd, Topographic Laser Ranging and Scanning: Principles and Processing. CRC Press: Boca Raton, Taylor \& Francis Group, FL, pp. 291-313.

ISO. 2005. ISO 9000 Quality management systems - fundamentals and vocabulary. Standard, International Organization for Standardization.

ISO. 2013. ISO 19157 Standard: Geographic information - data quality. Standard, International Organization for Standardization.

Jung, J., and Sohn, G. 2018. "Progressive modeling of 3D building rooftops from airborne Lidar and imagery.” In: Shan, J. and Toth, C.K. 2ndEd, Topographic Laser Ranging and Scanning: Principles and Processing. CRC Press: Boca Raton, Taylor \& Francis Group, FL, pp. 523-562.

Krämer, M., Haist, J., and Reitz, T. 2007. "Methods for spatial data quality of 3D city models." Eurographics Italian chapter conference, pp. 167-172. 
Kaartinen, H., Hyyppä, J., Gülch, E., Vosselman, G., Hyyppä, H., Matikainen, L., Hofmann, A.D. et al. 2005. "Accuracy of 3D city models: EuroSDR comparison." Int. Arch. Photogramm. Remote Sens. Spat. Inf. Sci. (ISPRS), vol. 36, pp. 227-232.

Ostrowski, W., Pilarska, M., Charyton, J., and Bakuła, K. 2018. “Analysis of 3D building models accuracy based on the airborne Laser scanning point clouds." Int. Arch of the Photogrammetry, Remote Sensing and Spatial Information Sciences, Volume XLII-2, ISPRS TC II Mid-term Symposium “Towards Photogrammetry 2020”, 4-7 June, Riva del Garda, Italy. https://doi.org/10.5194/isprs-archives-XLII-2-797-2018.

Park, S.Y., Lee, D.G., Yoo, E.J., and Lee, D.C. 2019. "Segmentation of Lidar data using multilevel cube code." Journal of Sensors, Volume 2019, Article ID 4098413, https://doi.org/10.1155/2019/4098413.

Rottensteiner, F., Sohn, G., Gerke, M., Wegner, J. D., Breitkopf, U., and Jung, J. 2014. "Results of the ISPRS benchmark on urban object detection and 3D building reconstruction." ISPRS Journal of Photogrammetry and Remote Sensing, 93(2014), 256-271.

Rottensteiner, F., and Clode, S. 2018. "Building and road extraction from Lidar data." In: Shan, J. and Toth, C.K. 2ndEd, Topographic Laser Ranging and Scanning: Principles and Processing. CRC Press: Boca Raton, Taylor \& Francis Group, FL, pp. 485-522.

Sampath, A., and Shan, J. 2010. "Segmentation and reconstruction of polyhedral building roofs from aerial Lidar point clouds." IEEE transactions on geoscience and remote sensing, vol. 48, no. 3, march 2010.

Shan, J., Yan, J., and Jiang, W. 2018. "Global solutions to building segmentation and reconstruction.” In: Shan, J. and Toth, C.K. 2ndEd, Topographic Laser Ranging and Scanning: Principles and Processing. CRC Press: Boca Raton, Taylor \& Francis Group, FL, pp. 459-484.

Stoter, J., Vallet, B., Lithen, T., Pla, M., Wozniak, P., Kellenberger, T., Streilein, A., Ilves, R., and Ledoux, H. 2016. "Stateof- the-art of 3D national mapping in 2016." Int. Arch. Photogramm. Remote Sens. Spatial Inf. Sci. XLI-B4, pp. 653-660.

Tarsha Kurdi, F. 2008. 3D building extraction and modelling from Lidar data. $\mathrm{PhD}$ grade at Photogrammetry and Geomatics Group MAP-PAGE UMR 694, Graduate School of Science and Technology of Strasbourg (INSA of Strasbourg) (ULP University).

Tarsha Kurdi, F., Landes, T., Grussenmeyer, P., and Koehl, M. 2007. "Model-driven and datadriven approaches using Lidar data: analysis and comparison." PIA07, Photogrammetry Image Analysis. 19 - 21 September 2007, Munich, Germany.

Tarsha Kurdi, F., Landes, T., and Grussenmeyer, P. 2008. "Extended RANSAC algorithm for automatic detection of building roof planes from Lidar data." The Photogrammetric Journal of Finland. Vol. 21, n ${ }^{\circ}, 2008$, pp.97-109. 
Tarsha Kurdi, F., Awrangjeb, M., and Munir, N. 2019. "Automatic 2D modelling of inner roof planes boundaries starting from Lidar data." 14th 3D GeoInfo 2019, 26-27 September 2019. Singapore, DOI: 10.5194/isprs-annals-IV-4-W8-107-2019.

Tarsha Kurdi, F., and Awrangjeb, M. 2020. "Automatic evaluation and improvement of roof segments for modelling missing details using Lidar data." International Journal of Remote Sensing, 41(12):4700-4723, https://doi.org/10.1080/01431161.2020.1723180.

Toth, C.K., and Koppanyi, Z. 2018. "Strip adjustment." In: Shan, J. and Toth, C.K. 2ndEd, Topographic Laser Ranging and Scanning: Principles and Processing. CRC Press: Boca Raton, Taylor \& Francis Group, FL, pp. 259-290.

Warchol, A. 2019. "The concept of Lidar data quality assessment in the context of BIM modeling." Int. Arch. Photogramm. Remote Sens and Spatial Information Sciences, Volume XLII-1/W2, Evaluation and Benchmarking Sensors, Systems and Geospatial Data in Photogrammetry and Remote Sensing, 16-17 Sept. Warsaw, Poland, https://doi.org/10.5194/isprs-archives-XLII-1-W2-61-2019, pp. 61-66.

Wehr, A., 2018. "Lidar systems and calibration.” In: Shan, J. and Toth, C.K. 2ndEd, Topographic Laser Ranging and Scanning: Principles and Processing. CRC Press: Boca Raton, Taylor \& Francis Group, FL, pp. 159-200.

Wehr, A., and Lohr, U. 1999. "Airborne laser scanning - an introduction and Overview." ISPRS Journal of Photogrammetry and Remote Sensing, 54, (2-3), pages 68-82.

Wong, K., and Ellul, C. 2016. "Using geometry-based metrics as part of fitness-for-purpose evaluations of 3D city models." ISPRS Annals of the Photogrammetry, Remote Sensing and Spatial Information Sciences, Volume IV-2/W1, 2016 11th 3D Geoinfo Conference, 20-21 October 2016, Athens, Greece.

Yilmaz, G., Akcamete, A., and Demirors, O. 2019. "A reference model for BIM capability assessments." Automation in Construction, 101 (2019), https://doi.org/10.1016/j.autcon.2018.10.022, pp. 245-263.

Zhang, K., Yan, J., and Chen, S.C. 2018. "A framework for automated construction of building models from airborne Lidar measurements." In: Shan, J. and Toth, C.K. 2ndEd, Topographic Laser Ranging and Scanning: Principles and Processing. CRC Press: Boca Raton, Taylor \& Francis Group, FL, pp. 563-586.

Zhao, J., Stoter, J. and Ledoux, H. 2014. "A Framework for the Automatic Geometric Repair of CityGML Models.” In: Buchroithner, M., Prechtel, N. and Burghardt, D. (eds) Cartography from Pole to Pole. Lecture Notes in Geoinformation and Cartography. Springer, Berlin, Heidelberg, pp 187-202. 